\title{
Real-Time Immersion of Physical Experiments in Virtual Wave-Physics Domains
}

\author{
Theodor S. Becker®, ${ }^{1,{ }^{*}}$ Nele Börsing $\odot,{ }^{1}$ Thomas Haag, ${ }^{1}$ Christoph Bärlocher, ${ }^{1}$ Carly M. Donahue, ${ }^{2}$ \\ Andrew Curtis ${ }^{3},{ }^{3}$ Johan O. A. Robertsson ${ }^{\circ},{ }^{1}$ and Dirk-Jan van Manen ${ }^{1}{ }^{1}$ \\ ${ }^{1}$ Institute of Geophysics, ETH Zürich, 8092 Zürich, Switzerland \\ ${ }^{2}$ Earth and Environmental Sciences, Los Alamos National Laboratory, Los Alamos, New Mexico 87545, USA \\ ${ }^{3}$ School of GeoSciences, University of Edinburgh, Edinburgh EH9 3FE, United Kingdom
}

(Received 4 February 2020; revised manuscript received 29 April 2020; accepted 11 May 2020; published 25 June 2020)

\begin{abstract}
We immerse a two-dimensional physical wave-propagation experiment in a virtual (simulated) environment in real time. This enables broadband hybrid wave experimentation in physical media that may be governed by unknown physics, embedded seamlessly within virtual media with either modeled or measured physics. Using the theory of immersive boundary conditions and a recently presented control system [Becker et al., Immersive Wave Propagation Experimentation: Physical Implementation and OneDimensional Acoustic Results, Phys. Rev. X 8, 031011 (2018)], we convert a circular two-dimensional acoustic waveguide into a larger physicovirtual square waveguide. Real-time wave-field extrapolation allows waves to propagate seamlessly between physical and virtual media, producing correct wavefield interactions between them, including nonlinear effects. We show that the laboratory can physically measure the response of nonphysical energy-gain materials in real time. The physicovirtual laboratory thus allows previously inaccessible wave phenomena to be investigated experimentally and constitutes an alternative approach for wave-physics investigations by connecting experimental and numerical approaches.
\end{abstract}

DOI: 10.1103/PhysRevApplied.13.064061

\section{INTRODUCTION}

In the era before digital computers, physical experiments in wave-propagation laboratories were crucial for the interpretation of physical observations, for testing scientific hypotheses, and for exploring complex wave physics (see, e.g., Refs. [1,2]). Since then, numerical modeling of virtual environments of ever-increasing complexity has become an essential element of hypothesis testing based on understood physical relationships (see, e.g., Refs. [3-5]). The role of physical laboratories is therefore mainly to explore novel or less well-understood physics or to investigate wave propagation in highly complex media that cannot easily be simulated [6-10]. However, such laboratories often suffer from unwanted back-scattering of waves from the physical boundary of the laboratory, which can mask waveforms of interest and hamper their interpretation [1]. This issue could be overcome by using shorter probing signals (higher frequencies) to obtain a time separation of the reflected waves and the signal of interest. However, since many wave phenomena are frequency dependent [11-13], such a scaling approach does not constitute a satisfying solution. Alternatively, the boundary of the laboratory can be rendered "invisible" for the probing

\footnotetext{
*theodor.becker@erdw.ethz.ch
}

wave field and reflections suppressed by the appropriate use of boundary conditions. This is, for instance, applied in anechoic chambers, commonly used for the calibration of microphones and loudspeakers, where a large volume of absorptive material is placed on the edges of a cavity to suppress reflections from its boundary [14]. A hybrid anechoic chamber has been suggested by Habault et al. [15], where the suppression of reflections for low frequencies is further improved by placing active control sources on the boundary, which inject a secondary wave field that destructively interferes with the boundary reflections.

Here, we take full control of the acoustic wave field on the boundary of a physical laboratory using densely spaced control sources. Not only does this allow us to virtually remove the influence of the boundary for broadband acoustic waves in a dynamic and flexible way but the physical laboratory can also be coupled to a surrounding virtual medium. This connects physical and virtual wavepropagation laboratories, immersing one within the other, thus creating a physicovirtual laboratory for wave-field experimentation. Virtual media with understood, hypothesized, or measured physical relations can be linked to any physical medium of arbitrary complexity to create a physicovirtual medium. The laboratory relies on the physical implementation of so-called immersive boundary conditions (IBCs) [16-18] to link the two media. Unlike 
existing experimental demonstrations of active wave-field control (see, e.g., Refs. [19-24]), the physicovirtual laboratory accounts for all orders of scattering interactions between physical and virtual media. Since the implemented boundary conditions are nonlocal in time and space, the laboratory allows spectrally and spatially broadband waves to propagate seamlessly between both media, without requiring a priori knowledge of the incident wave field. Measurements are made in real time in this laboratory and no assumptions are made about the underlying physics, so that complex nonlinear behavior and wavefield interactions with nonphysical virtual media can be observed. Thus, the physicovirtual laboratory allows the physical investigation of wave phenomena that can otherwise only be studied numerically. Moreover, it enables experimentation at significantly lower frequencies than is possible in conventional wave-propagation laboratories, which commonly suffer from adverse boundary reflections [1] or scaling-related issues [11-13].

We start by describing the concept of immersive wave experimentation (Sec. II). The two-dimensional (2D) acoustic laboratory and physicovirtual measurements from a nonphysical medium are presented in Sec. III. The ability of IBCs to reproduce all orders of wave-field interactions between physical and virtual objects is also demonstrated in this section. In Sec. IV, the broadband nature of the physicovirtual laboratory is demonstrated and the associated reduction in sound pressure levels (SPLs) and repeatability are discussed. Finally, further implications of the physicovirtual laboratory are presented in Sec. V.

\section{CONCEPT OF IMMERSIVE WAVE EXPERIMENTATION}

To create a physicovirtual laboratory, we consider the following question: "Are there boundary conditions that make wave propagation on one finite-volume domain behave like wave propagation on another domain?" Under certain conditions, it turns out that this can be achieved by using IBCs $[16,17]$. IBCs have previously been used primarily to truncate the spatial domain of numerical wavefield simulations while still producing wave fields from nontruncated simulations $[17,18]$. The theory of IBCs has been described in detail in previous work [16-18] and is briefly revisited in Appendix A with reference to acoustic wave fields (similar principles apply for elastic and electromagnetic wave propagation). Three processes immerse a physical domain of interest ( $\left.V_{\text {phys }}\right)$ within a surrounding virtual domain $\left(V_{\text {virt }}\right)$ by dynamically coupling the wave propagation in the two domains: (1) the acoustic wave fields are measured on a surface of sensors $S^{\text {rec }}$ near the edge of $V_{\text {phys }}$; (2) a Kirchhoff-Helmholtz integral is evaluated in real time using virtual-medium Green's functions to extrapolate the measured wave fields to the boundary

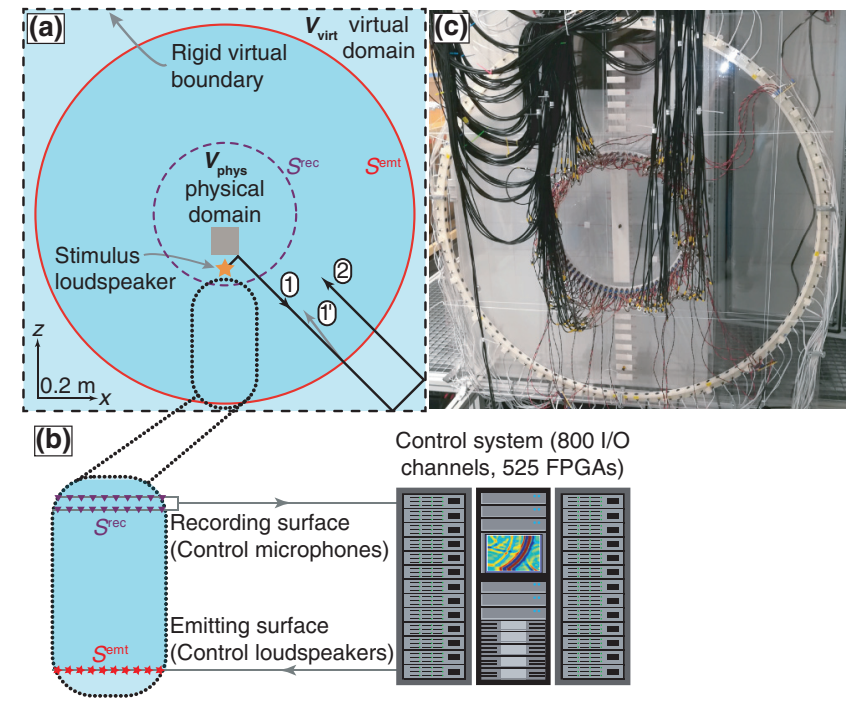

FIG. 1. A schematic illustrating the concept of experimentation using IBCs. (a) Waves incident on the boundary (1) of the physical domain are extrapolated from $S^{\text {rec }}$ (purple line) to all sources on $S^{\mathrm{emt}}$ (red line), priming those sources to emit energy such that reflections $\left(1^{\prime}\right)$ are suppressed. Wave-field interactions with the virtual domain are included in the extrapolation and emitted appropriately by the boundary sources (2). This also holds for higher-order scattering between the physical and virtual domains. The stimulus loudspeaker used to initiate individual experiments is shown as an orange star and the gray square represents a scatterer in the physical domain. The either acoustically transparent or rigid numerically simulated virtual boundary of $V_{\text {virt }}$ is denoted by a black dashed line. (b) Recordings from two dense surfaces of microphones on $S^{\text {rec }}$ (purple triangles) are fed to the low-latency control system, which extrapolates the wave field to $S^{\mathrm{emt}}$ in real time, where it is injected by the control loudspeakers (red stars). (c) A photograph of the 2D acoustic physicovirtual laboratory used in this study.

of the physical domain $\left(S^{\mathrm{emt}}\right)$ as well as through the virtual environment; and (3) the extrapolated wave field is applied as the signature of a set of secondary sources populating the boundary of the physical domain (Figs. 1 and 8). The secondary sources then act as perfect sinks for the primary field incident on the boundary, canceling all boundary reflections. If $V_{\text {virt }}$ is a heterogeneous medium, wave-field interactions with those heterogeneities are included in the Kirchhoff-Helmholtz extrapolation and radiate back into the physical domain from the secondary sources. This approach holds for any order of scattering interactions between the physical and virtual domain, including all wave phenomena arising from these interactions, and does not require a priori information about the incident wave field or the medium inside the recording surface.

The idea of using IBCs to couple a physical and a virtual or numerical domain in real time has been proposed by Vasmel et al. [25] and is referred to as immersive 
experimentation. One of the most challenging aspects of implementing IBCs physically arises from the need to predict the wave field around the entire boundary of the physical domain fast enough: the wave field needs to be known on the boundary before it physically arrives there. This is required to prime the signature of the secondary sources populating $S^{\mathrm{emt}}$ in order to cancel the arriving energy. That is, the set of all recordings of energy on $S^{\text {rec }}$ must be extrapolated computationally to each secondary source on $S^{\text {emt }}$ by evaluating Eq. (A3) at faster-than-real wavepropagation speed, so the total latency between physically recording the wave field on $S^{\text {rec }}$ and applying the required boundary conditions on $S^{\mathrm{emt}}$ needs to be smaller than the shortest physical travel time between the surfaces. This makes the design of the extrapolation and control system particularly challenging and as a result, to date only onedimensional (1D) immersive wave experimentation has been achieved, for which only a few control sources and receivers are required and the extrapolation reduces to a trivial sum [26-28]. To achieve immersive wave experimentation in higher dimensions, a tailor-made low-latency control system is constructed, which allows the extrapolation from 800 simultaneous analog input channels to 800 simultaneous analog output channels at a sample rate of $20 \mathrm{kHz}$ in no more than $200 \mu \mathrm{s}$. For more details on the architecture of the real-time data acquisition, computation, and control system, and on the numerical implementation and real-time evaluation of Eq. (A3), see Broggini et al. [18] and Becker et al. [26].

\section{REAL-TIME IMMERSION OF TWO-DIMENSIONAL ACOUSTIC EXPERIMENTS}

In the following, we present measurements made in a 2D acoustic physicovirtual laboratory, enabled by real-time IBCs acting on the boundary of a circular waveguide.

In this laboratory, a total of 156 acoustic sensors (microphones) are connected to 104 acoustic emitters (loudspeakers) through the control system to physically implement the required boundary conditions. In the experiments, reflections from the boundary of the laboratory are suppressed and all orders of wave-field interactions with a virtual simulated scattering domain surrounding the physical experiment are produced, thereby fully immersing the physical experiment within the virtual background. The experimental setup is described in detail in Appendix B and a schematic and photograph are provided in Fig. 1.

It is worth noting that in addition to the computationally demanding wave-field extrapolation, the physical implementation of IBCs involves a range of real-time hardware corrections to comply with the theory of IBCs. These corrections include the removal of sensor and emitter transfer functions and particle-velocity estimation from pressure recordings, as well as a correction for the imperfect domain boundary. These corrections are discussed in detail in Appendix C and also in Becker et al. [26].

To demonstrate the physicovirtual laboratory, we first turn the edge of the experiment from a physical circle into a physicovirtual square. In a first experiment without boundary control (IBCs disabled), a Ricker wavelet (i.e., a wavelet described by the second derivative of a Gaussian function [29]) with a dominant frequency $\left(f_{d}\right)$ of $3 \mathrm{kHz}$ is emitted into the waveguide by a loudspeaker placed at $(0,-0.2) \mathrm{m}$ [see Fig. 2(a)]. The resulting outwardpropagating pressure wave is recorded by the two circular microphone arrays between around 1.3 and $2.5 \mathrm{~ms}$ [label 1 in Fig. 2(a)]. The wave reflects from the circular boundary of the waveguide, propagates inward, and is again recorded by the microphones between 3.6 and $5.3 \mathrm{~ms}$ (label $2)$; it then propagates outward again, creating a reverberating field (label 3 and subsequent events). The same

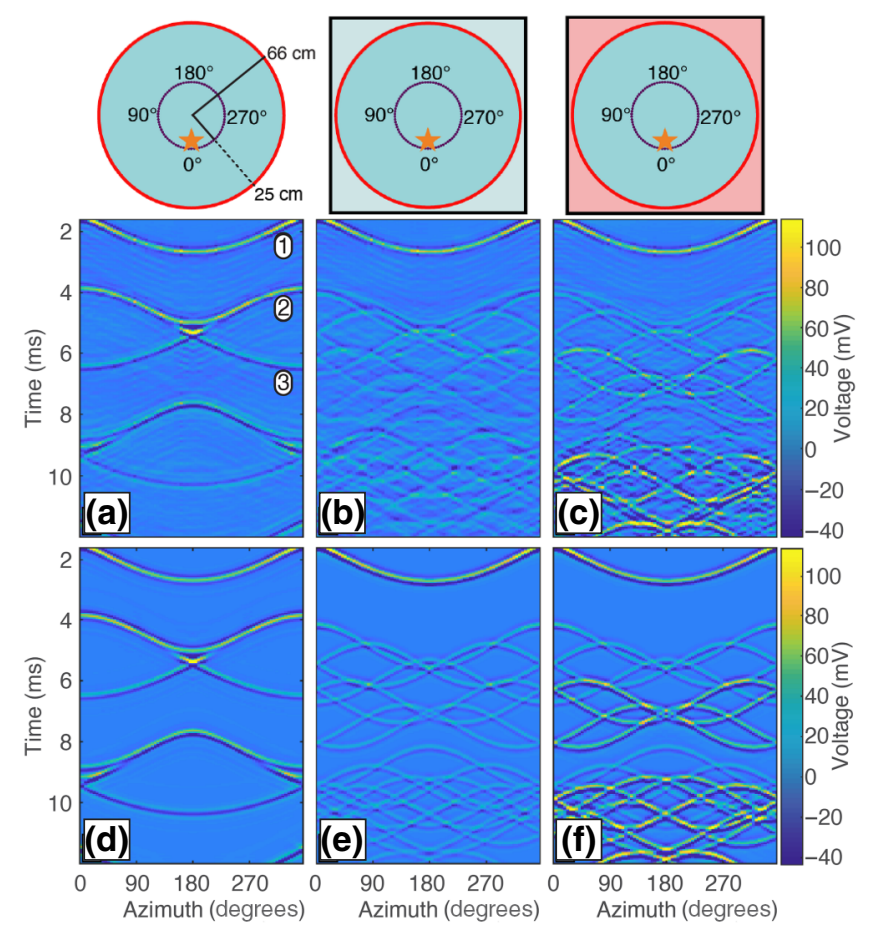

FIG. 2. Results demonstrating physicovirtual experiments in a 2D circular acoustic waveguide, which is immersed in a virtual square domain. The respective setups are sketched in the top row (notation as in Fig. 1). (a)-(c) Experimental data extracted at the outer circular microphone array without (a) and with (b),(c) active boundary control using IBCs. (d)-(f) Numerical simulations of the acoustic wave fields computed on a circular (d) and square (e),(f) model with perfectly reflecting boundaries. The virtual domain outside the waveguide is lossless for the experiments shown in (b) and (e), while the virtual medium is energy gaining for the results in (c) and (f) (represented by the light blue and red color in the top sketches). Labels 1-3 are explained in the main text. Events recorded after around $8 \mathrm{~ms}$ have interacted with the virtual domain at least twice. 
experiment is repeated with IBCs enabled [Fig. 2(b)]. The virtual domain is chosen to be square, with side lengths of $1.4 \mathrm{~m}$ and perfectly rigid boundaries, in which Green's functions used for real-time extrapolation are modeled (the black dashed line in Fig. 1 thus represents the rigid outer boundary of the virtual domain). This time, the broadband reflection of the wave incident on $S^{\text {emt }}$ is strongly suppressed (with a reduction in SPL of approximately $12 \mathrm{~dB}$ ), rendering the physical boundary transparent for broadband acoustic signals. Moreover, all wave-field reflections from the virtual rigid boundary of the square background medium are reintroduced into the physical waveguide, including long-range multiple interactions with the virtual rigid boundary. The comparison with numerical simulations [Figs. 2(d) and 2(e)] confirms that when IBCs are used, the physical measurements in the waveguide closely match the wave propagation in a square medium, even though the measurements are made in a circular physical domain. Thus, the circular physical waveguide is fully immersed in a larger square physicovirtual medium in real time. For the previous experiment, the virtual domain consists of a lossless material. In a second IBC-enabled experiment, the square virtual domain surrounding the physical waveguide consists of an energy-gaining medium with a frequency-independent gain of $13 \mathrm{~dB} / \mathrm{m}$. The experimental results and a corresponding numerical simulation are displayed in Figs. 2(c) and 2(f). The wave energy increases as waves travel through the gaining medium, leading to elevated wave amplitudes at later times because those events have probed a larger area of the gaining material. Since such energy-gaining media do not occur naturally, this experiment demonstrates the ability to physically measure the response of nonphysical media in the physicovirtual laboratory.

\section{A. Immersive wave experimentation including higher-order scattering}

To show that real-time immersive wave experimentation also encompasses higher-order long-range scattering interactions between physical and virtual scatterers, a $10 \times 10 \mathrm{~cm}$ aluminum block is placed in the physical waveguide and the immersive wave experiments repeated. Note that for these experiments, the extrapolation Green's functions are identical to those in the previous experiment (without the aluminum block present). Changes of the medium within $S^{\text {rec }}$ do not require an update of the extrapolation Green's functions [30,31]; thus no prior knowledge of the medium properties within $S^{\text {rec }}$ is required.

The resulting complex acoustic wave field contains events that have interacted multiple times with both physical and virtual domains [Fig. 3(a)]. To highlight the relatively weak multiply scattered interactions, Fig. 3(b) shows the difference between wave fields with and without

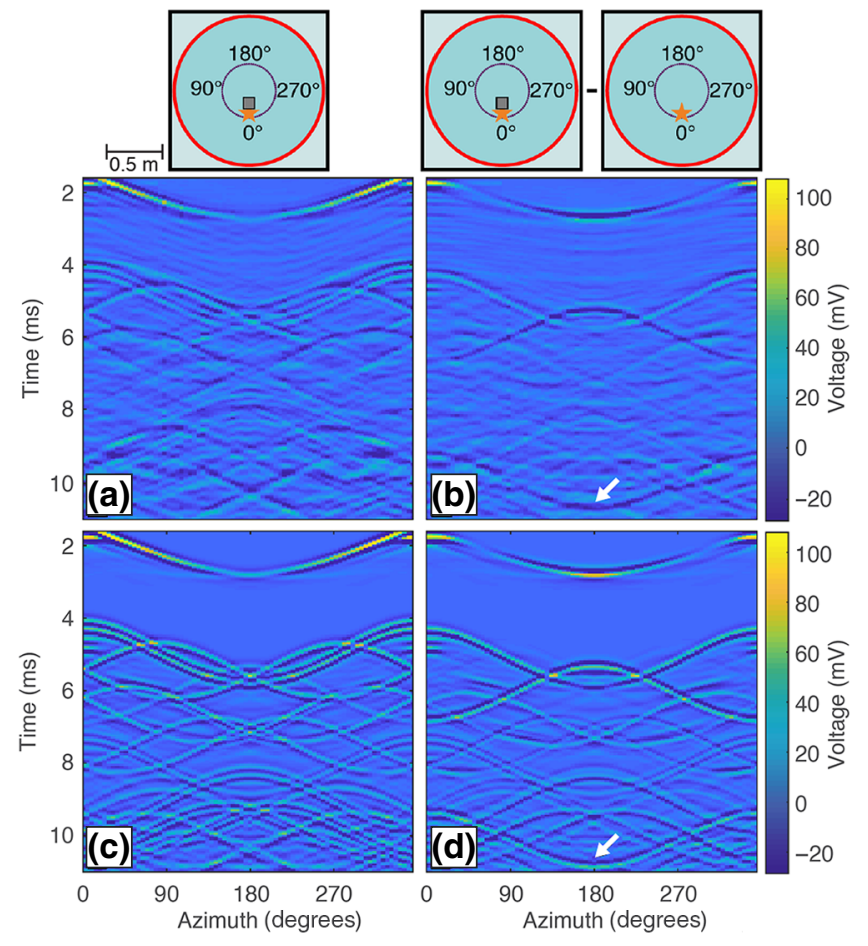

FIG. 3. Results of full wave-field immersion including higherorder long-range physicovirtual scattering. The top row shows sketches of the experimental setup (notation as in Fig. 1). (a),(b) Experimental data extracted at the outer circular microphone array with active boundary control enabled. (c),(d) Numerical simulations of the pressure field computed on a square model with perfectly reflecting boundaries. Panels (a) and (c) show the pressure field with the aluminum scatterer present in $V_{\text {phys }}$ (gray square in top row). The differences of the wave fields with and without the scatterer (i.e., the wave-field components due to the scatterer) are displayed in (b) and (d). Events recorded after approximately $7 \mathrm{~ms}$ represent multiply scattered physicovirtual interactions, e.g., a forward-scattered event that reflected at the top and bottom boundaries of the virtual domain, which propagated three times through the physical domain in between (white arrows).

the aluminum block, which are compared with numerical simulations on a square domain with and without a $10 \times 10 \mathrm{~cm}$ rigid scatterer [Figs. 3(c) and 3(d) and 4]. Of particular interest are those events observed after approximately $7 \mathrm{~ms}$, which represent multiply scattered interactions between physical and virtual scatterers. Despite the complexity and low amplitudes of the scattered field due to geometrical spreading, the experimental results contain wave fields comparable to the reference simulations (with a normalized root-mean-square error of about $10.0 \%$ ), demonstrating the ability of immersive wave experimentation to reproduce long-range higher-order interactions. Thus, we may perform experiments and make recordings in the physical laboratory as though they are conducted in the virtually extended domain. 

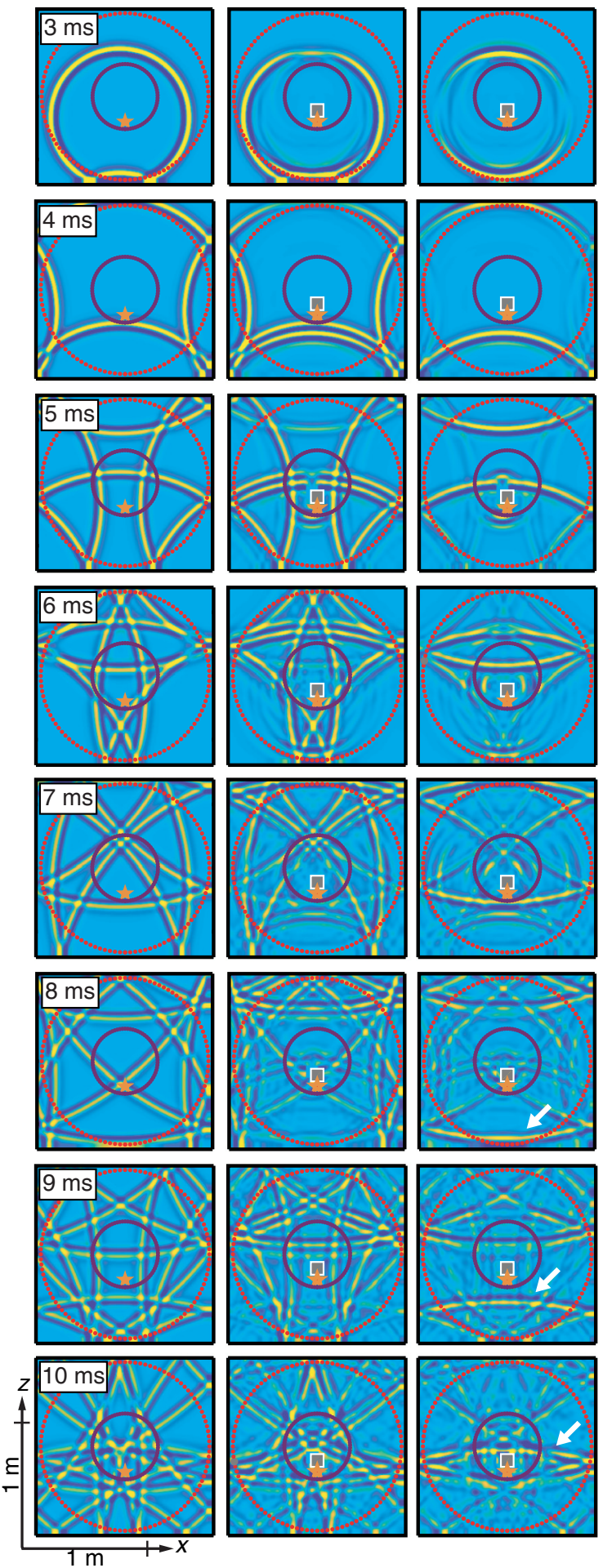

FIG. 4. Snapshots of the numerical simulations without (left) and with (center) the scatterer in $V_{\text {phys }}$, respectively. The right column shows the difference between the middle and left columns, i.e., the field that is due to the physical scatterer (gray square). Orange star, stimulus loudspeaker; purple circle, outer circular microphone array; red dots, secondary loudspeakers on $S^{\mathrm{emt}}$; black boundary, the perfectly rigid boundary in the numerical simulations. The white arrows show the same event highlighted in Fig. 3.

\section{DISCUSSION}

We demonstrate that the physical implementation of IBCs enables the construction of physicovirtual laboratories in which waves can propagate seamlessly between physical and virtual media. The only condition on the physicovirtual medium is that the properties and physics are the same in both domains between the recording and emitting surface (i.e., the physical and virtual domains overlap). This does not imply that the physics or medium in this region must be known, since the virtual medium can also be represented by a set of measured (instead of modeled) extrapolation Green's functions. In that case, no knowledge whatsoever of the medium parameters and physics of either the virtual or physical domains is required. In the following, we demonstrate the broadband nature of immersive wave experimentation and discuss the repeatability of immersive wave experiments.

\section{A. Broadband nature of immersive wave experimentation}

Compared to other control strategies, particularly those relying on passive materials, which are often effective only for very narrow frequency bands [32,33], immersive boundary conditions are both spatially and spectrally broadband and hence capable of suppressing boundary reflections across a wide frequency band and for

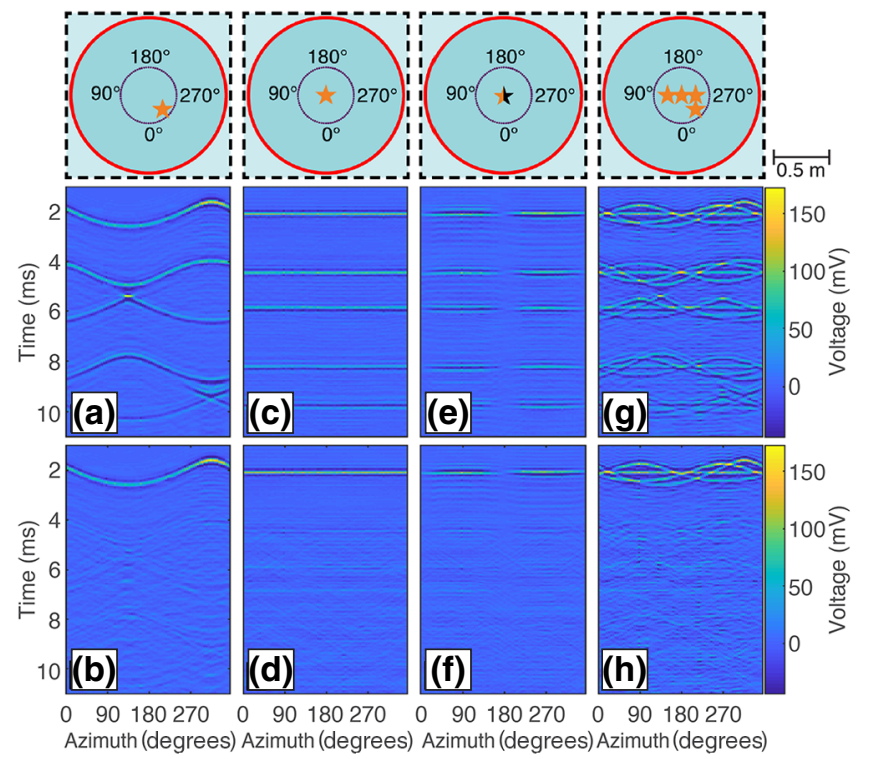

FIG. 5. Experiments demonstrating the broadband nature of immersive wave experimentation. The experimental setup is sketched in the top row, where the dashed black line represents an acoustically transparent boundary and the orange-black star an effective dipole source. The rest of the symbol key follows that of Fig. 1. Recordings on the outer microphone array of $S^{\text {rec }}$ without and with active boundary control using IBCs are shown in the second and third row, respectively. 
varying source locations. We experimentally demonstrate the broadband nature of IBCs by carrying out four different experiments with varying source locations and signatures, without making changes to the control algorithm or hardware. This also illustrates that no a priori knowledge of the incident wave fields is required. The four experiments are: (1) a Ricker wavelet with $f_{d}=3 \mathrm{kHz}$ emitted at $(0.12,-0.12) \mathrm{m}$ [Figs. 5(a) and 5(b)], (2) a stimulus loudspeaker emitting a Ricker wavelet with $f_{d}=4 \mathrm{kHz}$ at the center of the waveguide [Figs. 5(c) and 5(d)], (3) an effective horizontal dipole source emitting a Ricker wavelet with $f_{d}=4 \mathrm{kHz}$ at the plate center [Figs. 5(e) and 5(f)], and (4) four loudspeakers simultaneously emitting Ricker wavelets with $f_{d}=4 \mathrm{kHz}$ at $(-0.2,0) \mathrm{m}$, $(0,0) \mathrm{m},(0.2,0) \mathrm{m}$, and $(0.12,-0.12) \mathrm{m}$ [Figs. $5(\mathrm{~g})$ and $5(\mathrm{~h})]$. Note that for these experiments, no scatterers are present in $V_{\text {phys }}$ or $V_{\text {virt }}$ (so that the black dashed line in Fig. 1 represents a transparent boundary), thus only the direct acoustic wave is extrapolated from $S^{\text {rec }}$ and emitted on $S^{\text {emt }}$. Despite the increasing complexity of the emitted wave fields, all boundary reflections are successfully suppressed in the four experiments, thereby effectively rendering the boundary transparent for broadband acoustic
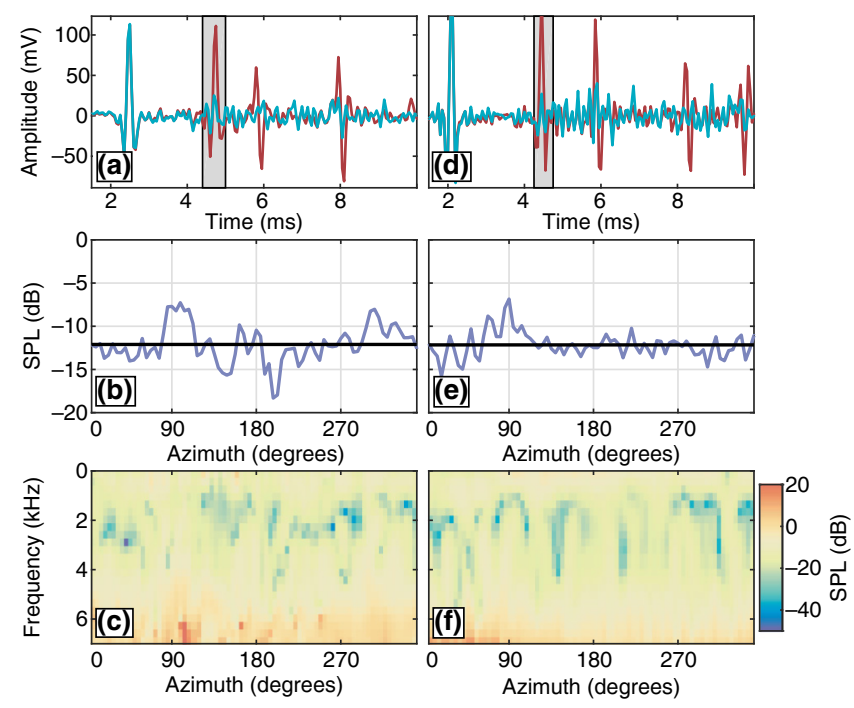

FIG. 6. The time series and SPLs for the leftmost two experiments in Fig. 5. (a),(d) The voltage recorded with a microphone at an azimuth of approximately $184^{\circ}$ without (red) and with (blue) active boundary control. The gray boxes denote the time windows around the first boundary reflection used for the calculation of the reduction in SPL when boundary control is enabled (b),(e). The horizontal black lines represent the mean SPL of $-12.1 \mathrm{~dB}$ (left) and -12.2 dB (right). (c),(f) A frequency-domain representation of the reduction in SPL within the gray boxes, with a reduction in mean reflected SPL of more than $10 \mathrm{~dB}$ over a frequency range from approximately $400 \mathrm{~Hz}$ and $4400 \mathrm{~Hz}$ for the first experiment (off-center source, $f_{d}=3 \mathrm{kHz}$ ) and from approximately $900 \mathrm{~Hz}$ to $5300 \mathrm{~Hz}$ for the second experiment (central source, $f_{d}=4 \mathrm{kHz}$ ).

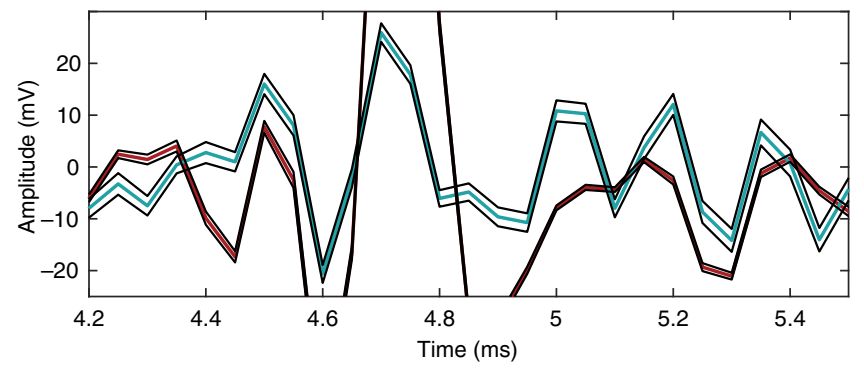

FIG. 7. The mean and standard deviation of 20 realizations of the leftmost experiment of Fig. 5. The close-ups of the time series recorded at an azimuth of approximately $184^{\circ}$ are centered around the first boundary reflection. The mean values for 20 experiments without and with boundary control are displayed in red and blue, respectively, and the standard deviation is shown in black.

signals and immersing the physical experiment in a homogeneous half-space.

To further quantify the ability of IBCs to suppress broadband boundary reflections, the leftmost two experiments of Fig. 5 are considered in more detail. Figures 6(a) and 6(d) display example time series without (red) and with (blue) active boundary control for these experiments. Moreover, Figs. 6(b), 6(c), 6(e), and 6(f) show the reduction in SPL in the first boundary reflection with enabled boundary control using IBCs. As expected, the SPL is significantly reduced across all 78 microphones of the outer microphone array on $S^{\text {rec }}$ for both experiments, with a reduction of more than $10 \mathrm{~dB}$ over approximately 3.7 octaves.

\section{B. Repeatability of immersive wave experiments}

The leftmost experiment of Fig. 5 is repeated 20 times to analyze the repeatability of immersive wave experimentation. Figure 7 shows 20 realizations of microphone recordings at an azimuth of approximately $184^{\circ}$. It is evident that the standard deviation of the pressure recordings is significantly lower than the signal level and also lower than the residual energy in the suppressed reflected waves. This indicates that incoherent acoustic or electronic noise does not significantly contribute to the resulting time series and that most residual energy can be attributed to coherent source-generated noise.

\section{CONCLUSIONS}

The physicovirtual laboratory enables the study of complex wave propagation in materials governed by unknown physics under arbitrary boundary conditions (e.g., radiation or periodic boundary conditions). Such investigations are not realizable in purely numerical simulations, because unknown physics cannot easily be represented in a computer without a model, but also not in a conventional wave-propagation experiment, for which the boundary conditions cannot be dynamically adapted. The ability to 
apply real-time boundary conditions that turn full multiply scattered wave propagation in one domain into propagation in another domain has fundamental and unexpected consequences that allow previously experimentally inaccessible phenomena in wave physics to be investigated. Since measurements are made in real time in this physicovirtual laboratory, no assumptions about the underlying physics are made and also complex nonlinear behavior or wave-field interactions with nonphysical virtual media can be measured. For instance, we demonstrate experimentally that responses from virtual energy-gaining material can be observed in the physicovirtual laboratory. The capability to exactly balance a physical loss material with a well-described virtual gain material could be exploited to build acoustic super-resolution focusing devices and unidirectional transparency by creating parity-time-symmetric (PTS) acoustic media [34,35], which thus far has been limited to the numerical realm or 1D media due to the inability to realize energy-gaining materials in higher dimensions. Apart from PTS media, the physicovirtual laboratory constitutes a stepping stone for the study of periodic metamaterials through the use of periodic boundary conditions on the virtual domain: waves leaving the laboratory on one side enter the laboratory on the opposite side. In this way, the physical laboratory and scatterers placed within it can be considered as the unit cell of an infinitely periodic lattice, leading to a range of unusual and exotic wave phenomena [36]. When interchanging the recording and emitting surfaces of the physicovirtual laboratory, the presented methodology can also be used for broadband acoustic cloaking and holography [32,37-40], as has been presented in 1D experiments in Börsing et al. [27]. This could be used to conceal objects from acoustic waves or to create acoustic illusions in real time and for arbitrary incident wave fields. Finally, as a listener or observer in a medium or cavity surrounded by IBCs, one would not be able to discern whether they were in the larger physical medium or any one of an infinite set of smaller media with IBCs applied. The ability to switch off reflections from the boundary of the laboratory also implies that the frequency and wavelength limitations associated with experimentation in the original smaller volume are replaced by frequency and wavelength limits associated with the larger physicovirtual medium. This property enables the physics of wave propagation to be studied at significantly lower frequencies than in conventional laboratories without undesired boundary reflections masking the signal of interest. Hence, when scaled to three dimensions (3D), the presented methodology could, for instance, be used to (re)produce complex real (or imagined) immersive acoustic environments for listeners in a cavity enclosed by IBCs [41-43]. Moreover, a 3D physicovirtual laboratory would enable audio-hardware calibration under arbitrary boundary conditions; for example, loudspeaker-response measurements in a simulated anechoic chamber or rooms with various tunable acoustic characteristics. While the scaling to $3 \mathrm{D}$ is nontrivial, it is one of our key current objectives and the control system enabling physicovirtual experimentation, as described in Becker et al. [26], is built and designed for a full-scale 3D laboratory.

\section{ACKNOWLEDGMENTS}

We thank four anonymous reviewers for their comments and constructive feedback and Badreddine Assouar for editorial handling. Furthermore, T.S.B. wishes to thank Emily Rose Ciscato, Patrick Elison, Henrik Rasmus Thomsen, and Xun Li for discussions, reviewing, and feedback. This work was funded by the European Union's Horizon 2020 research and innovation programme under the Marie Sklodowska-Curie Grant Agreement No. 641943 and has also received funding from the European Research Council (ERC) under the European Union's Horizon 2020 research and innovation programme (Grant Agreement No. 694407).

Our contributions to this work are as follows: data curation and formal analysis, T.S.B.; funding acquisition, J.O.A.R. and D.M.; investigation, T.S.B. and N.B.; methodology, D.M., A.C., J.O.A.R., N.B., and T.S.B.; resources and software, T.H., C.B., N.B., T.S.B., and C.M.D.; supervision, D.M., J.O.A.R., A.C., and C.M.D.; validation and visualization, T.S.B.; writing, T.S.B., A.C., D.M., and J.O.A.R.

\section{APPENDIX A: THEORY OF IMMERSIVE BOUNDARY CONDITIONS}

In the following, we briefly summarize the derivation of the IBCs for an acoustic physicovirtual laboratory. More details on IBCs can be found in previous publications [16-18]. To this end, we denote by $V_{\text {phys }}$ the physical acoustic laboratory to be fully immersed in $V_{\text {virt }}$, an arbitrarily complex virtual domain surrounding the physical laboratory (see Fig. 8). In this study, $V_{\text {virt }}$ is represented by numerical simulations but it could also be represented by a set of measured medium responses.

The physical boundary of the laboratory is assumed to be rigid (sound hard) and is denoted by $S^{\text {emt }}$. Without active boundary control on $S^{\mathrm{emt}}$, waves reflect back from this boundary into the interior of $V_{\text {phys }}$ (top part of Fig. 8). It is our goal to render $S^{\text {emt }}$ virtually transparent for acoustic waves and to fully couple the wave propagation in $V_{\text {phys }}$ and $V_{\text {virt }}$ by injecting secondary sources on the boundary of the laboratory with appropriate source signatures. This state, for which $V_{\text {phys }}$ is fully immersed in $V_{\text {virt }}$ and waves can propagate seamlessly back and forth, is denoted by $V_{\text {full }}$ (bottom part of Fig. 8). Intuitively, the necessary boundary conditions to be injected on $S^{\text {emt }}$ can be found by differencing the pressure-field representations in $V_{\text {full }}$ and $V_{\text {phys }}$, which are denoted by $p_{\text {full }}(\mathbf{x}, t)$ and $p_{\text {phys }}(\mathbf{x}, t)$, respectively. Using the reciprocity theorem 

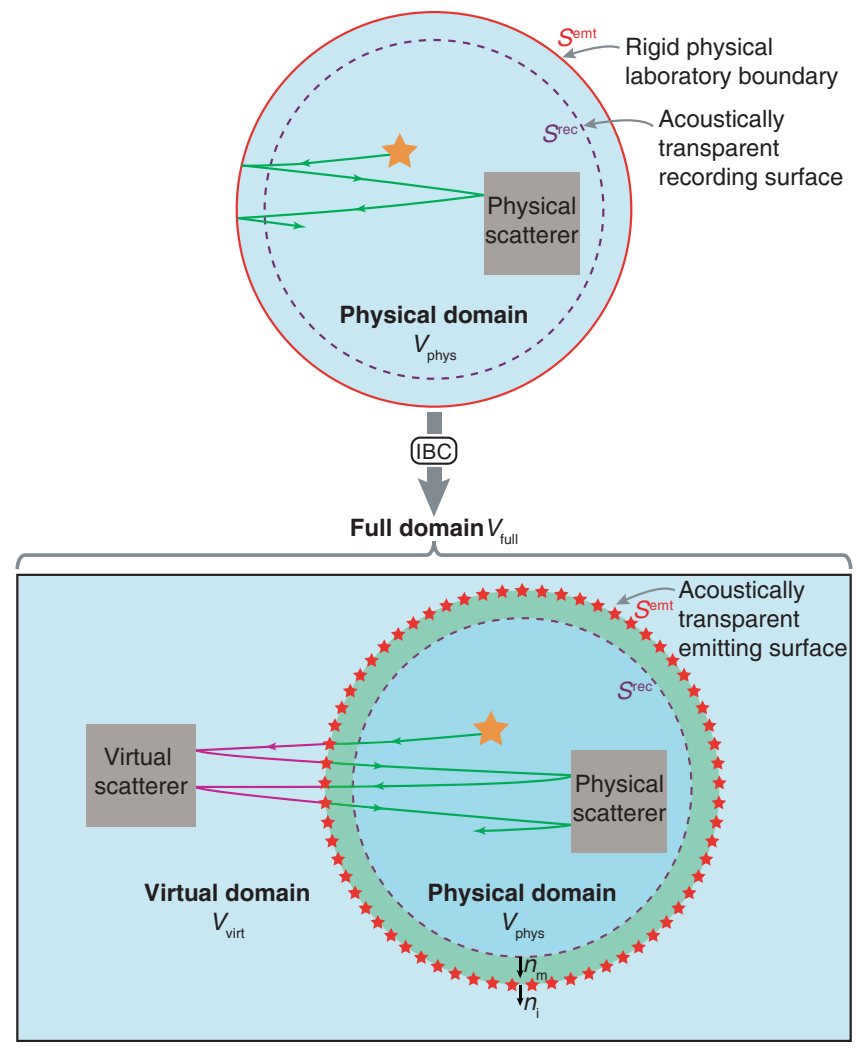

FIG. 8. A schematic illustrating the concept and domains of IBCs. The physical laboratory is denoted by $V_{\text {phys }}$ and bounded by a rigid surface $S^{\mathrm{emt}}$ (top part of the figure). Through the injection of IBCs by secondary sources on $S^{\mathrm{emt}}$ (red stars in the bottom part of the figure), the boundary is rendered acoustically transparent and waves can seamlessly propagate between the physical laboratory (green arrows) and the surrounding virtual domain (purple arrows). This state, for which $V_{\text {phys }}$ is fully immersed in $V_{\text {virt }}$, is denoted by $V_{\text {full }}$. The green area in the bottom part of the figure represents the common region of $V_{\text {phys }}$ and $V_{\text {virt }}$, for which the medium parameters need to be identical in both domains.

of the convolution type, this difference can be expressed as [18]

$$
\begin{aligned}
& p_{\text {full }}\left(\mathbf{x}^{\prime}, t\right)-p_{\text {phys }}\left(\mathbf{x}^{\prime}, t\right) \\
& =-\oint_{S^{\text {emt }}}\left[G_{\text {phys }}^{p, q}\left(\mathbf{x}^{\prime}, \mathbf{x}, t\right) * v_{\text {full }, i}(\mathbf{x}, t)\right] n_{i} d S, \\
& \quad \text { for } \mathbf{x}^{\prime} \in V_{\text {phys }},
\end{aligned}
$$

where temporal convolution is represented by an asterisk, $G_{\text {phys }}^{p, q}\left(\mathbf{x}^{\prime}, \mathbf{x}, t\right)$ is the acoustic pressure impulse response (Green's function) of the medium at $\mathbf{x}^{\prime}$ due to a point source of volume injection rate volume density at $\mathbf{x}$, $v_{\text {full }, i}(\mathbf{x}, t)$ is the $i$ th component of the particle velocity in the full domain, and $n_{i}$ denotes the $i$ th component of the outward-directed normal on $S^{\text {emt }}$. Note that for the derivation of Eq. (A1), $S^{\mathrm{emt}}$ is assumed to be a rigid boundary, but a similar expression can be derived for a free surface at $S^{\mathrm{emt}}$
[18]. The normal particle velocity on $S^{\text {ent }}$ can be obtained by using a Kirchhoff-Helmholtz integral to extrapolate the wave fields from an auxiliary recording surface $S^{\text {rec }}$ inside $V_{\text {phys }}$ to $S^{\mathrm{emt}}[16,30]$ :

$$
\begin{aligned}
v_{\mathrm{full}, i}\left(\mathbf{x}^{\mathrm{emt}}, t\right)= & \oint_{S^{\mathrm{rec}}}\left[G_{\mathrm{fuul}, i}^{v, q}\left(\mathbf{x}^{\mathrm{emt}}, \mathbf{x}, t\right) * v_{\mathrm{full}, m}(\mathbf{x}, t)\right. \\
& \left.+G_{\text {full }, i, m}^{v, f}\left(\mathbf{x}^{\mathrm{emt}}, \mathbf{x}, t\right) * p_{\mathrm{full}}(\mathbf{x}, t)\right] n_{m} d S .
\end{aligned}
$$

Here, $G_{\text {full }, i}^{v, q}\left(\mathbf{x}^{\mathrm{emt}}, \mathbf{x}, t\right)$ and $G_{\text {full,i, }, \text { v }}^{v, f}\left(\mathbf{x}^{\mathrm{emt}}, \mathbf{x}, t\right)$ represent the $i$ th component of the particle-velocity impulse response of $V_{\text {full }}$ due to a monopole source and an $m$-directed dipole source, respectively, and $n_{m}$ is the $m$ th component of the outward-pointing normal on $S^{\mathrm{rec}}$. To facilitate the implementation of Eq. (A2) on the real-time control system, we rewrite the equation recursively and discretize in time $[16,18]$ :

$$
\begin{aligned}
\hat{v}_{\text {full }, i}\left(\mathbf{x}^{\mathrm{emt}}, k ; l\right)= & \hat{v}_{\text {full }, i}\left(\mathbf{x}^{\mathrm{emt}}, k-1 ; l\right) \\
& +\oint_{S^{\mathrm{rec}}}\left[\hat{G}_{\text {full }, i}^{v, q}\left(\mathbf{x}^{\mathrm{emt}}, \mathbf{x}, l-k\right) \hat{v}_{\text {full }, m}(\mathbf{x}, k)\right. \\
& \left.+\hat{G}_{\text {full }, i, m}^{v, f}\left(\mathbf{x}^{\mathrm{emt}}, \mathbf{x}, l-k\right) \hat{p}_{\text {full }}(\mathbf{x}, k)\right] n_{m} d S,
\end{aligned}
$$

where time-discretized quantities are represented by ${ }^{\wedge}$, and $k$ and $l$ are the discrete time index and discrete lag time index, respectively (with $k, l \in \mathbf{N} \mid 1 \leq k, l \leq N_{t}$, where $N_{t}$ is the total number of time steps in an experiment). Equations (A1) and (A3) form the core for the implementation of IBCs on $S^{\text {emt }}$. The two equations can be interpreted as follows: $V_{\text {phys }}$ is fully immersed into $V_{\text {virt }}$ by emitting monopole sources on $S^{\mathrm{emt}}$, which are scaled with the normal particle velocity on $S^{\mathrm{emt}}$ measured in $V_{\text {full }}$ [Eq. (A1)]. Clearly, $\hat{v}_{\text {full }, i}\left(\mathbf{x}^{\mathrm{emt}}, k ; l\right) n_{i}$ must be predicted ahead of time (i.e., knowledge of the wave field arriving at the boundary of the truncated domain is required before it actually arrives). This is achieved by measuring the pressure and normal particle velocity on the auxiliary recording surface $S^{\text {rec }}$ and extrapolating both wave-field quantities to $S^{\text {emt }}$ [Eq. (A3)]. More precisely, at each time step $k$, the pressure and normal-particle-velocity fields with $l \geq k$ are extrapolated, $\hat{v}_{\text {full, } i}\left(\mathbf{x}^{\mathrm{emt}}, k ; l=k\right) n_{i}$ is applied as the source strength of the monopole sources on $S^{\text {emt }}$, and future values of $\hat{v}_{\text {full, }, i}\left(\mathbf{x}^{\mathrm{emt}}, k ; l>k\right) n_{i}$ are summed to a buffer for use at later times of the computation. The necessity to extrapolate the wave field from $S^{\text {rec }}$ to $S^{\text {emt }}$ is the reason why a region common to $V_{\text {phys }}$ and $V_{\text {virt }}$ exists in which the medium properties of both domains ought to be identical (green region in Fig. 8); everywhere else, they may differ. The required size of this region is determined by the latency of the extrapolation. 


\section{APPENDIX B: EXPERIMENTAL SETUP}

IBCs are physically implemented in an air-filled acoustic waveguide consisting of two parallel polymethyl methacrylate (PMMA) plates with a spacing of $a=2.5 \mathrm{~cm}$ (see Figs. 1 and 9). The wave propagation in the waveguide can be regarded as two-dimensional for signals below the cutoff frequency of the fundamental mode, $f_{c}=$ $0.5 \mathrm{ca}^{-1}$ [44], where $c$ is the speed of sound. This leads to $f_{c} \approx 6.9 \mathrm{kHz}$ for our experiments in air. The $2 \mathrm{D}$ character of wave propagation in the waveguide is experimentally validated by comparing physical measurements to $2 \mathrm{D}$ simulations for various source-receiver locations [45]. According to the theory, the implementation of IBCs requires measurements of the pressure and normal particle velocity on $S^{\text {rec }}$ (see Sec. II). Instead of directly recording the particle velocity, the wave-field quantity can be derived from the gradient of the pressure field using two (or more) microphones oriented slightly inside and outside of $S^{\text {rec }}$, respectively. Hence, for our experiments the recording surface comprises two circular arrays with radii of $23.8 \mathrm{~cm}$ and $25.8 \mathrm{~cm}$, respectively, each consisting of 78 electret condenser microphones. The microphones are mounted flush with the inside of one of the PMMA plates, and are separated by $d=2 \mathrm{~cm}$ in the normal direction. The boundary of the experimental domain, which coincides with $S^{\text {emt }}$, consists of a circular 3D printed array with a radius of $66.2 \mathrm{~cm}$. The array houses 104 loudspeakers spaced $4 \mathrm{~cm}$ in the tangential direction. For experiments in air, the distance of approximately $41 \mathrm{~cm}$ between $S^{\text {rec }}$ and $S^{\text {emt }}$ allows a maximum total latency of about $1200 \mu \mathrm{s}$, of which $200 \mu$ s is used for the extrapolation and the remaining $1000 \mu \mathrm{s}$ is available for hardware corrections and the

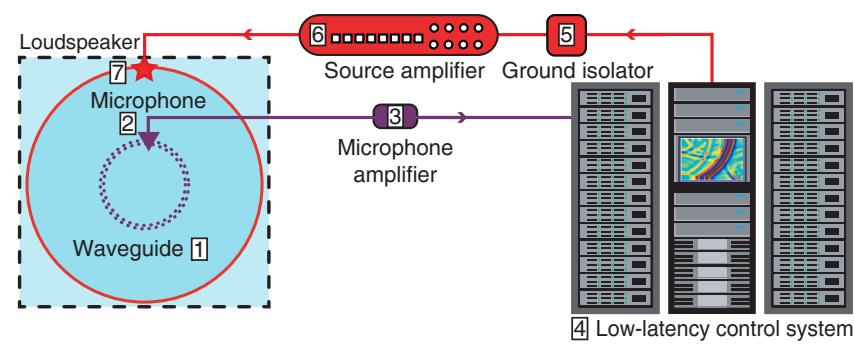

FIG. 9. The hardware chain used for 2D acoustic immersive wave experimentation: 1, waveguide consisting of two parallel PMMA plates with a $2.5-\mathrm{cm}$ separation distance; 2 , electret condenser microphone (SparkFun electronics, COM-08635) on $S^{\text {rec }}$; 3 , electret microphone amplifying circuit (SparkFun electronics, BOB-12758), modified with a $470 \Omega$ resistor and a $2.2 \mu \mathrm{F}$ capacitor; 4, low-latency control system consisting of 800 analog-input and 800 analog-output channels; 5, ground isolator (Monacor, FGA-40MF); 6, eight-channel $50 \mathrm{~W}$ class-D digital amplifier (Monacor STA-850D); 7, $8 \Omega, 1.2 \mathrm{~W}$ loudspeaker (PUI Audio, AS01508MR-R) on $S^{\mathrm{emt}}$. real-time estimation of the normal particle velocity from pressure recordings.

\section{APPENDIX C: MODELING AND MANIPULATION OF THE EXTRAPOLATION GREEN'S FUNCTIONS}

In theory, the implementation of IBCs requires closely spaced particle velocity and pressure measurements on $S^{\text {rec }}$ to be extrapolated to $S^{\text {emt }}$, where the extrapolated wave fields are injected as the signatures of closely spaced monopole sources on a rigid boundary. However, "perfect" measurements of the pressure and particle velocity, monopolar sources, and perfectly rigid boundaries are idealized theoretical constructs that are difficult to realize in a physical experiment. Hence, a physical implementation of IBCs requires a range of hardware-related corrections to be made in order to comply with the theory. For our experiments, we consider scalar and frequency-dependent corrections only, which are represented by short temporal matched filters a few coefficients in length. Due to the associative property of the convolution, these corrections can be made as part of the real-time extrapolation by manipulating the extrapolation Green's functions in Eq. (A3) prior to an experiment. This has the advantage that no additional computations or filtering operations are needed at run time. Recently, it has been found that a similar approach also holds for angle-dependent corrections, such as corrections for the nonmonopolar directivity pattern of the control loudspeakers [46].

For the experiments described in this work, the creation and manipulation of the extrapolation Green's functions consists of the following steps:

(1) Forward modeling of the extrapolation Green's functions from each microphone on $S^{\text {rec }}$ to each loudspeaker on $S^{\text {emt }}$ using 2D acoustic finite-difference modeling with second-order accuracy in time and space.

(2) Application of a $0.3-10 \mathrm{kHz}$ bandpass filter.

(3) Removal of the loudspeaker and amplifier transfer functions by convolving the Green's functions with matched filters that represent the inverse transfer functions of each loudspeaker-amplifier pair.

(4) Removal of the microphone transfer functions by scaling the Green's functions with the microphone sensitivities.

(5) For $\hat{G}_{\text {full, }, i}^{v, q}\left(\mathbf{x}^{\mathrm{emt}}, \mathbf{x}, l-k\right)$, estimating the particle velocity from two staggered pressure recordings based on the equation of motion; for $\hat{G}_{\mathrm{full}, i, m}^{v, f}\left(\mathbf{x}^{\mathrm{emt}}, \mathbf{x}, l-k\right)$, linear interpolation of two staggered pressure recordings to the midpoint between the microphones (for details, see Becker et al. [26]).

(6) Application of matched filters (one per control loudspeaker) to $\hat{G}_{\text {full,i,m }}^{v, f}\left(\mathbf{x}^{\mathrm{emt}}, \mathbf{x}, l-k\right)$ that optimize the wavefield separation implied in Eq. (A3). The matched filters 
are determined in an initial calibration experiment with homogeneous $V_{\text {phys }}$ and $V_{\text {virt }}$ and a stimulus source placed at the plate center. Hence, only a single event should be extrapolated to $S^{\mathrm{emt}}$. The matched filters are then obtained by minimizing the residual energy following the direct extrapolated wave. Note that this approach has similarities to earlier work for the decomposition of marine seismic wave-field data [47].

(7) Decomposition of the Green's functions into a direct part and a part due to scatterers in $V_{\text {virt }}$, so that a correction for the deviation from a perfectly rigid boundary at $S^{\text {emt }}$ can be applied to the direct part of the Green's function only. In a calibration experiment, a single filter is estimated that matches the wave field resulting from the emission of the control loudspeakers (with all previously described corrections applied) to the reflected wave field resulting from the emission of a stimulus loudspeaker at the plate center. This filter is then applied to the direct part of the extrapolation Green's function and represents the frequency-dependent (normal-incidence) reflection coefficient of the physical boundary at $S^{\mathrm{emt}}$ but also accounts for any residual errors from the removal of hardware transfer functions.

More details on the manipulation of the extrapolation Green's functions are provided in earlier work $[26,46]$.

[1] P. N. S. O’Brien and M. P. Symes, Model seismology, Rep. Prog. Phys. 34, 697 (1971).

[2] J. A. McDonald, G. H. F. Gardner, and F. J. Hilterman, in Seismic Studies in Physical Modeling, edited by John A. McDonald (IHRDC Press, Boston, 1983), 1st ed..

[3] Jean Virieux, $P-S V$ wave propagation in heterogeneous media: Velocity-stress finite-difference method, Geophysics 51, 889 (1986).

[4] Christopher K. W. Tam and Jay C. Webb, Dispersionrelation-preserving finite difference schemes for computational acoustics, J. Comput. Acoust. 107, 262 (1993).

[5] Andreas Fichtner, Full Seismic Waveform Modelling and Inversion, 1st ed., Advances in Geophysical and Environmental Mechanics and Mathematics (Springer, Berlin, Heidelberg, 2011).

[6] R. A. Shelby, D. R. Smith, and S. Schultz, Experimental verification of a negative index of refraction, Science 292, 77 (2001).

[7] Roel Snieder, Alexandre Grêt, Huub Douma, and John Scales, Coda wave interferometry for estimating nonlinear behavior in seismic velocity, Science 295, 2253 (2002).

[8] Vincent Bacot, Matthieu Labousse, Antonin Eddi, Mathias Fink, and Emmanuel Fort, Time reversal and holography with spacetime transformations, Nat. Phys. 12, 972 (2016).

[9] Bence Solymosi, Nathalie Favretto-Cristini, Vadim Monteiller, Dimitri Komatitsch, Paul Cristini, Børge Arntsen, and Bjørn Ursin, How to adapt numerical simulation of wave propagation and ultrasonic laboratory experiments to be comparable - A case study for a complex topographic model, Geophysics 83, T195 (2018).

[10] N. Favretto-Cristini, B. Solymosi, P. Cristini, V. Monteiller, B. Ursin, and D. Komatitsch, in OCEANS 2019-Marseille (Marseille, France, 2019), p. 1.

[11] Grant A. Gist, Interpreting laboratory velocity measurements in partially gas-saturated rocks, Geophysics 59, 1100 (1994).

[12] T. Cadoret, D. Marion, and B. Zinszner, Influence of frequency and fluid distribution on elastic wave velocities in partially saturated limestones, J. Geophys. Res. 100, 9789 (1995).

[13] Michael L. Batzle, De-Hua Han, and Ronny Hofmann, Fluid mobility and frequency-dependent seismic velocity_Direct measurements, Geophysics 71, N1 (2006).

[14] Leo L. Beranek and Harvey P. Sleeper, The design and construction of anechoic sound chambers, J. Acoust. Soc. Am. 18, 140 (1946).

[15] D. Habault, E. Friot, Ph. Herzog, and C. Pinhede, Active control in an anechoic room: Theory and first simulations, Acta Acust. United Acust. 103, 369 (2017).

[16] Dirk-Jan van Manen, Johan O. A. Robertsson, and Andrew Curtis, Exact wave field simulation for finite-volume scattering problems, J. Acoust. Soc. Am. 122, EL115 (2007).

[17] Marlies Vasmel, Ph.D. thesis, ETH Zürich, Switzerland (2016).

[18] Filippo Broggini, Marlies Vasmel, Johan O. A. Robertsson, and Dirk-Jan van Manen, Immersive boundary conditions: Theory, implementation, and examples, Geophysics 82, T97 (2017).

[19] E. Friot and C. Bordier, Real-time active suppression of scattered acoustic radiation, J. Sound Vib. 278, 563 (2004).

[20] Hervé Lissek, Romain Boulandet, and Romain Fleury, Electroacoustic absorbers: Bridging the gap between shunt loudspeakers and active sound absorption, J. Acoust. Soc. Am. 129, 2968 (2011).

[21] R. Boulandet and H. Lissek, Toward broadband electroacoustic resonators through optimized feedback control strategies, J. Sound Vib. 333, 4810 (2014).

[22] E. Friot, A. Gintz, P. Herzog, and S. Schneider, in Motion and Vibration Control, edited by Heinz Ulbrich and Lucas Ginzinger (Springer, Dordrecht, 2011), p. 83.

[23] Etienne Rivet, Sami Karkar, and Hervé Lissek, On the optimisation of multi-degree-of-Freedom acoustic impedances of low-frequency electroacoustic absorbers for room modal equalisation, Acta Acust. United Acust. 103, 1025 (2017).

[24] Shuping Wang, Jingxia Yu, Xiaojun Qiu, Marek Pawelczyk, Abu Shaid, and Lijing Wang, Active sound radiation control with secondary sources at the edge of the opening, Appl. Acoust. 117, 173 (2017).

[25] Marlies Vasmel, Johan O. A. Robertsson, Dirk-Jan van Manen, and Andrew Curtis, Immersive experimentation in a wave propagation laboratory, J. Acoust. Soc. Am. 134, EL492 (2013).

[26] Theodor S. Becker, Dirk-Jan van Manen, Carly M. Donahue, Christoph Bärlocher, Nele Börsing, Filippo Broggini, Thomas Haag, Johan O. A. Robertsson, Darren R. Schmidt, Stewart A. Greenhalgh, and Thomas E. Blum, Immersive Wave Propagation Experimentation: Physical Implementation and One-Dimensional Acoustic Results, Phys. Rev. X 8, 031011 (2018). 
[27] Nele Börsing, Theodor S. Becker, Andrew Curtis, DirkJan van Manen, Thomas Haag, and Johan O. A. Robertsson, Cloaking and Holography Experiments Using Immersive Boundary Conditions, Phys. Rev. Appl. 12, 024011 (2019).

[28] Henrik R. Thomsen, Miguel Molerón, Thomas Haag, Dirk-Jan van Manen, and Johan O. A. Robertsson, Elastic immersive wave experimentation: Theory and physical implementation, Phys. Rev. Res. 1, 033203 (2019).

[29] Yanghua Wang, Frequencies of the Ricker wavelet, Geophysics 80, A31 (2015).

[30] J. T. Fokkema and P. M. van den Berg, Seismic Applications of Acoustic Reciprocity (Elsevier, Amsterdam, 1993), 1st ed.

[31] C. J. Thomson, Research note: Internal/external seismic source wavefield separation and cancellation, Geophys. Prospect. 60, 581 (2012).

[32] Romain Fleury, Francesco Monticone, and Andrea Alù, Invisibility and Cloaking: Origins, Present, and Future Perspectives, Phys. Rev. Appl. 4, 037001 (2015).

[33] Steven A. Cummer, Johan Christensen, and Andrea Alù, Controlling sound with acoustic metamaterials, Nat. Rev. Mater. 1, 16001 (2016).

[34] Chengzhi Shi, Marc Dubois, Yun Chen, Lei Cheng, Hamidreza Ramezani, Yuan Wang, and Xiang Zhang, Accessing the exceptional points of parity-time symmetric acoustics, Nat. Commun. 7, 1 (2016).

[35] Romain Fleury, Dimitrios L. Sounas, and Andrea Alu, Parity-time symmetry in acoustics: Theory, devices, and potential applications, IEEE J. Sel. Top. Quantum Electron. 22, 121 (2016).

[36] Dirk-Jan van Manen, Miguel Moleron, Henrik Rasmus Thomsen, Nele Börsing, Theodor S. Becker, Michael R. Haberman, and Johan O. Robertsson, Immersive boundary conditions for meta-material experimentation, J. Acoust. Soc. Am. 146, 2786 (2019).
[37] S. Ise, A principle of sound field control based on the Kirchhoff-Helmholtz integral equation and the theory of inverse systems, Acustica 85, 78 (1999).

[38] Emmanuel Friot, Régine Guillermin, and Muriel Winninger, Active control of scattered acoustic radiation: A real-time implementation for a three-dimensional object, Acta Acust. United Acust. 92, 278 (2006).

[39] Dirk-Jan van Manen, Marlies Vasmel, Stewart Greenhalgh, and Johan O. A. Robertsson, Broadband cloaking and holography with exact boundary conditions, J. Acoust. Soc. Am. 137, EL415 (2015).

[40] Daniel Eggler and Nicole Kessissoglou, Active acoustic illusions for stealth and subterfuge, Sci. Rep. 9, 1 (2019).

[41] A. J. Berkhout, D. de Vries, and P. Vogel, Acoustic control by wave field synthesis, J. Acoust. Soc. Am. 93, 2764 (1993).

[42] Jens Ahrens and Sascha Spors, An analytical approach to sound field reproduction using circular and spherical loudspeaker distributions, Acta Acust. United Acust. 94, 988 (2008).

[43] Jordan Cheer, Active control of scattered acoustic fields: Cancellation, reproduction and cloaking, J. Acoust. Soc. Am. 140, 1502 (2016).

[44] Martin Redwood, Mechanical Waveguides: The Propagation of Acoustic and Ultrasonic Waves in Fluids and Solids with Boundaries (Pergamon Press, New York, 1960), p. 57.

[45] Theodor Simon Becker, Ph.D. thesis, ETH Zürich, Switzerland (2020).

[46] Xun Li, Johan Robertsson, Andrew Curtis, and Dirk-Jan van Manen, Compensating for source directivity in immersive wave experimentation, J. Acoust. Soc. Am. 146, 3141 (2019).

[47] Aslaug H. S. Melbø, Johan O. A. Robertsson, and Dirk-Jan van Manen, in Society of Exploration Geophysicists 72nd Annual Meeting, Technical Program Expanded Abstracts (Society of Exploration Geophysicists, Salt Lake City, 2002), p. 1030. 